\title{
ESCLARECIMIENTO TAXONÓMICO DE NYMPHAEA GRACILIS ZUCC., PLANTA ACUÁTICA ENDÉMICA DE MÉXICO*
}

\author{
ALEJANDRO NOVELO R. y ANTONIO LOT H.**
}

\section{RESUMEN}

Se presenta una recopilación cronológica de Nymphaea ampla (Salisb.) DC., desde su descripción realizada por Salisbury en 1805 hasta la actualidad, mencionando algunos nombres que incorrectamente le han sido referidos como sinónimos, entre los que destaca el de Nymphaea gracilis Zucc.

$N$. gracilis por diversas características morfológicas y de comportamiento, al menos más evidentes en el campo, fácilmente se diferencia de $N$. ampla, la única otra especie mexicana con la cual se podría confundir, entre las cuales sobresalen: el borde de la hoja, forma del rizoma, las nervaduras del envés de las hojas, ángulo de abertura de los sépalos y el rango altitudinal.

Por los resultados emanados de la presente investigación, quedó evidenciado que N. ampla var. speciosa (Mart. \& Zucc.) Casp. por el momento, continúa restringida al arco antillano y a Sudamérica y que la confusión a que había dado lugar el trabajo de Conard (1905) al ampliar la distribución de esta variedad a las partes templadas de México, debido a la inclusión de $N$. gracilis y $N$. undulata bajo esta variedad, queda aclarada.

La comparación de las descripciones y de los ejemplares tipo de los nombres de varias ninfáceas que estaban inmiscuidos en el problema, como eran: $N$. gracilis Zucc., $N$. flavo-virens Lehm., $N$. undulata Lehm., y $N$. tussilagifolia Lehm., nos demostró, que todas ellas pertenecen a la misma especie y que por principio de prioridad, el nombre correcto que debe ser usado para esta planta es $N$. gracilis Zucc.

\section{ABSTRACT}

A review of the taxonomic history of Nymphaea ampla (Salisb.) DC. is presented,

*Recibido para publicación en julio de 1983.

** Departamento de Botánica, Instituto de Biología, Universidad Nacional Autónoma de México. Ap. Postal 70-233. 04510 México, D.F. 
since its description by Salisbury in 1805 up to the present, including the mention of various names which have been incorrectly referred to this taxon as synonyms, most notably Nymphaea gracilis Zucc.

$N$. gracilis is easily distinguished from $N$. ampla, the only other Mexican species with which it might be confused, by several morphological and field characters, namely: the margin of the leaf, shape of the rhizome, venation of the lower leaf surface, angle of aperture of the sepals, and altitudinal distribution.

The results of this research lead us to the conclusion that $N$. ampla var. speciosa (Mart. \& Zucc.) Casp. is restricted at present to the Antilles and South America. The confusion created by Conard's work (1905) widening the range of this variety to temperate Mexico, by the inclusion of $N$. gracilis and $N$. undulata under this variety, has been clarified.

Comparison of descriptions and type specimens of various Nymphaeas, which were involved in the problem, such as $N$. gracilis Zucc., $N$. flavo-virens Lehm., $N$. undulata Lehm., and N. tussilagifolia Lehm., indicates that all of them belong to the same species. Applying the rule of priority, the correct name which should be used for this plant is $N$. gracilis Zucc.

\section{INTRODUCCIÓN}

El género Nymphaea contiene aproximadamente 40 especies ampliamente distribuidas en el mundo. De éstas, México comparte siete, tanto de afinidad templada como tropical.

El estudio taxonómico de este importante grupo de plantas acuáticas, ha estado relegado desde 1905, año en el cual fue publicada la revisión taxonómica del género a nivel mundial por Conard. Hoy en día, urge una actualización total del género, principalmente en aquéllas áreas con una elevada diversidad como lo son las partes bajas y cálidas del sur de México, Centro y Sudamérica, así como el Caribe.

De las 12 especies pertenecientes al subgénero Brachyceras, que se caracteriza principalmente por presentar los estilos carpelares cortos, rígidos, carnosos y de forma ligulada y el ápice de los estambres con el conectivo muy desarrollado formando un apéndice puntiagudo, 3 se encuentran exclusivamente en el continente americano: Nymphaea ampla, $N$. elegans y $N$. gracilis.

Nymphaea ampla (Salisb.) DC. es la especie más común de los ambientes dulceacuícolas del trópico de nuestro continente, distribuyéndose desde el sur de Florida hasta el sur de Brasil, incluyendo el arco antillano. En México se ha colectado en los estados de Jalisco, Colima, Michoacán, Guerrero, Oaxaca y Chiapas, en la vertiente del Pacífico y en Coahuila, Nuevo León, Tamaulipas, San Luis Potosí, Veracruz, Puebla, Tabasco, Campeche, Yucatán y Quintana Roo, en la vertiente del Golfo.

$N$. elegans Hook. presenta una área de distribución menor, restringida al noreste y noroeste de México y sureste de Estados Unidos. En México se ha colectado en los estados de: Sonora, Sinaloa, Nayarit, Michoacán, Guerrero y Tamaulipas.

Finalmente, la especie que nos concierne en el presente trabajo, $N$. gracilis Zucc. es la que presenta una distribución más restringida y es endémica de las partes templadas del altiplano, centro y suroeste de México. Se ha colectado en los estados de Durango, Aguascalientes, Jalisco, Guanajuato, Michoacán, México, Distrito Federal y Oaxaca (Fig. 1). 


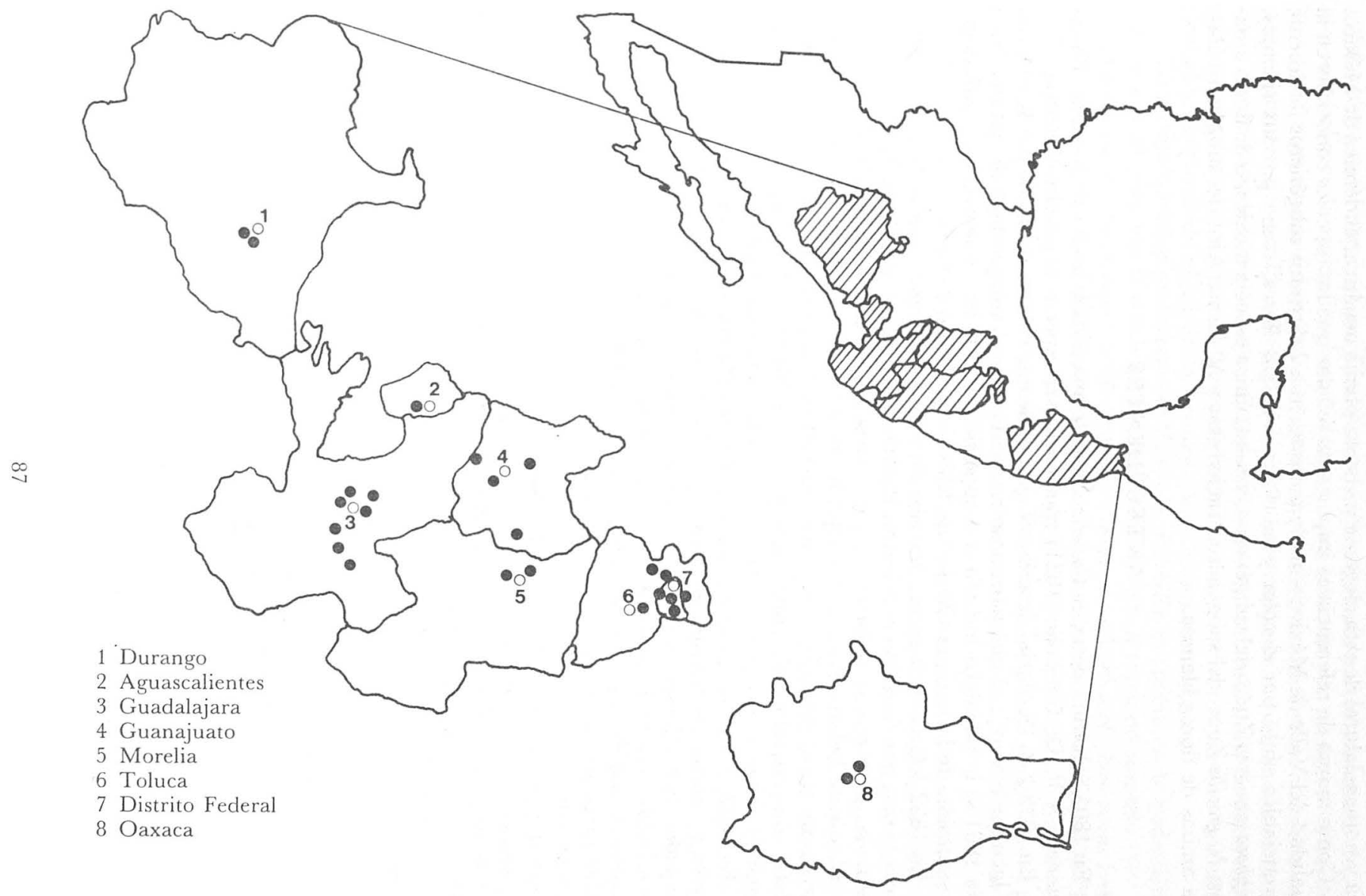

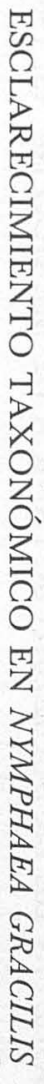

Fig. 1. Mapa de México en donde se indica la distribución de Nymphaea gracilis Zucc. 
El interés de la presente contribución, es aclarar y actualizar la situación taxonómica y nomenclatural de esta elegante y vistosa planta acuática, endémica de México.

Como marco de referencia es importante señalar que las especies conocidas en la actualidad del Valle de México son 3 y pertenecen a 2 distintos subgéneros fácilmente diferenciables tanto por el color y morfología de sus flores, como por sus rizomas: Nymphaea mexicana Zucc. del subgénero Castalia, única especie mexicana de flores amarillas; $N$. gracilis Zucc. del subgénero Brachyceras y $N$. odorata Ait., del subgénero Castalia ambas de flores blancas.

\section{ANTECEDENTES}

En 1805 Salisbury describe Castalia ampla de una colecta hecha en Jamaica. Posteriormente A.P. De Candolle (1821) transfiere esta especie al género Nymphaea.

En 1832 J.A. Zuccarini describe $N$. gracilis de una colecta realizada por Karwinski "in lacu mexicano", el que para nosotros parece ser el antiguo lago de México, que hacia 1861 se le calculaba todavía una superficie de $230 \mathrm{~km}^{2}$ y cubría una considerable extensión de la antigua Ciudad de México y sus alrededores.

En 1852 J.G.C. Lehmann, describe Nymphaea flavo-virens, según Hooker (1901), de una planta que floreció en el Jardín Botánico de Hamburgo, de procedencia indeterminada, pero que se sospechaba que podría haber sido llevada de México.

El mismo Lehmann en la descripción, la situaba cerca de $N$. gracilis, de la cual difería muy poco, principalmente en el tamaño de sus partes florales y vegetativas, y de ésta, con certeza se sabía que era originaria de México. Hasta ese momento, tanto $N$. gracilis como $N$. flavo-virens eran consideradas como dos especies diferentes.

En 1853, Planchon en su extenso estudio sobre la familia Nymphaeaceae hace un balance y análisis de las especies conocidas. En el caso de $N$. ampla, la divide en tres variedades, la $\alpha$ Plumieri que se distribuye en la región caribeña insular, citando ejemplares de Martinica y Jamaica; $\beta$ Hookeri que se distribuye tanto en la región continental como insular de América tropical y en la cual incluye a $N$. flavo-virens Lehm. como un sinónimo, aclarando que lo hace solamente juzgando la descripción, sin mencionar ningún ejemplar mexicano. Por último la variedad $\gamma$ Salzmanii oriunda del Brasil.

Es importante mencionar por otro lado, que Planchon en este trabajo, considera a $N$. gracilis Zucc. como una buena especie y distinta de $N$. ampla, citando para México además, los ejemplares colectados por Berlandier 145, Anúı ieux 354, 450 y Galeotti 4846, este último, colectado a $1524 \mathrm{~m}$ en el estado de Oaxaca.

En ese mismo año, Lehmann describe dos especies más, la primera, $N$. undulata basada en el ejemplar colectado por Galeotti procedente de Oaxaca, mencionado anteriormente y $N$. tussilagifolia, citada también por Planchon en su trabajo como Nymphaea sp. nova (p. 56) de un ejemplar colectado por Andrieux (Núm. 546) "In lacu Chalaensi [lago de Chalco] prope Yotla [cerca de Ayotla]", del Valle de México.

Planchon en el caso de $N$. tussilagifolia, por "l'imperfection des exemplaires" no tomó la decisión de darle un nombre, pero sí la sitúa por las características de los estambres, en la misma sección en donde ahora está colocada $N$. gracilis.

Posteriormente en 1878, cuando Caspary - profundo conocedor del género Nymphaea - colabora en la Flora Brasiliensis, avanza en el conocimiento y entendimiento principalmente de las secciones más complejas y diversas, en particular del trópico americano y en el cual más adelante, Conard se apoyaría para publicar su magna obra.

Este autor, también considera tres variedades para $N$. ampla, la variedad $\alpha$ Plumie- 
$r i$ (propuesta anteriormente por Planchon) distribuida en la parte continental de América tropical y en la región insular caribeña, y caracterizada por presentar el borde de las hojas irregularmente sinuoso - dentado, con los dientes agudos; las otras dos variedades consideradas por Planchon ( $\beta$ Hookeri y $\gamma$ Salzmanii) en cambio, quedan incluidas en la nueva variedad speciosa, que se distribuye en el arco antillano y Sudamérica, la cual se caracteriza por tener el borde de las hojas más o menos repando - sinuado a dentado, pero cuando el borde es dentado, los dientes son obtusos.

La última variedad propuesta por Caspary, Pulchella, es endémica de Sudamérica. Esta variedad presenta el borde de las hojas sub-entero a repando-sinuado.

Es muy importante hacer notar aquí que Caspary excluyó a $N$. flavo-virens Lehm. de $N$. ampla var. speciosa, debido probablemente a que este autor a diferencia de Planchon, además de basarse en la descripción de $N$. flavo-virens debió haber visto el ejemplar tipo y considerar que esta especie se salía del área principal de distribución, permitiéndole diferenciarla muy claramente de la variedad speciosa, a pesar de que las hojas de estas dos especies son muy parecidas.

Sin embargo, como $N$. flavo-virens Lehm. no quedaba considerada en la diagnosis para esa flora, Caspary no puso en claro totalmente su situación taxonómica.

En 1890 Pringle, uno de los colectores extranjeros de plantas mexicanas más activos de esa época, publica un trabajo sobre ninfáceas de nuestro territorio, en el cual reconoce cuatro especies: $N$. mexicana de flores amarillas, $N$. elegans de flores azules (más bien lilas) y $N$. ampla y $N$. gracilis, ambas de flores blancas. $N$. ampla la menciona principalmente de la parte norte del altiplano de México, desde Nuevo León hasta Texas, sobresaliendo esta especie por sus grandes hojas de color púrpura en el envés y venas prominentes, y por sus rizomas gruesos y largos. $N$. gracilis la reconoce de la vasta región irrigada por el Río Lerma, extendiéndose desde Aguascalientes y San Luis Potosí en el norte, hasta cerca de la ciudad de México en el este y sur, y más allá de Guadalajara en el oeste. Menciona que su tallo se asemeja más a un tubérculo que a un rizoma, al menos en otoño, después de que el resto de la planta ha decaído, quedando solamente un cuerpo ovoide de 1 a $5 \mathrm{~cm}$ de longitud con gruesa y dura cáscara cubierta con protuberancias que le dan la apariencia de un cono de pino.

A principios de siglo, Conard (1905) publica el más importante y único trabajo monográfico del género Nymphaea a un plano mundial, conocido a la fecha. La variedad Plumieri propuesta por Planchon y mantenida en uso por Caspary, Conard descarta el nombre de la variedad, aceptando y manteniendo el nombre específico, para la forma originalmente descrita (lo que actualmente sería $N$. ampla var. ampla). Esta variedad es la que presenta la distribución más extensa, desde Florida hasta Brasil, así como las Antillas.

Las dos variedades reconocidas por Conard, corresponden exactamente a las propuestas por Caspary en Flora Brasiliensis, la var. speciosa y la var. pulchella. La que nos interesa aquí es la var. speciosa por lo que nos extenderemos un poco más adelante, y de la var. pulchella solo diremos que ha sido colectada en Puerto Rico, Perú y Brasil.

En el caso de la variedad speciosa, Caspary la consideró propia de la región insular caribeña y de sudamérica, pero Conard amplía su distribución a México, al incluir en esta variedad como sinónimos a varias especies de ninfáceas descritas previamente de México y que se asemejan a esta variedad exclusivamente por el tipo de borde de la hoja, que va de más o menos repando-sinuado a dentado, pero con los dientes obtusos y los nervios de la parte inferior de la hoja ligeramente prominentes.

Específicamente menciona como sinónimos a $N$. gracilis Zucc. citando el ejemplar 
de Karwinski del Valle de México y $N$. undulata Lehm. citando el correspondiente ejemplar de Galeotti de Oaxaca; además cita como colectas adicionales, la 3891 de Pringle, de las cercanías de Guadalajara identificada originalmente como N. gracilis Zucc.

Por otro lado considera a $N$. flavo-virens Lehmann como una buena especie, diferente de $N$. ampla, pero sin saber con certeza el origen y distribución de esta planta. Menciona también que "An examination of Zuccarini's types shows this name to be mistaken, while the plants agree well with the types of $N$. flavo-virens both of Lehmann and Hooker.".

En este momento Conard, parece no darse cuenta de que $N$. gracilis fue descrita en 1832 mientras que $N$. flavo-virens no fue descrita sino hasta el año de 1852 y que por el principio de prioridad el nombre correcto para esta especie debería ser $N$. gracilis.

Posteriormente, Conard se pregunta a sí mismo, "Is $N$. flavo-virens Lehm. merely a cultivated form of $N$. gracilis Zucc. ( = N. ampla DC.)?". Esta frase es altamente significativa en el sentido de que Conard tuvo serias dudas con esta especie, dejándole al lector o estudioso, un serio problema de decisión en el que están inmiscuidos por lo menos tres nombres.

Conard, creemos nosotros, pudo resolver el asunto al momento de escribir la monografía, dado que él contaba con la información publicada unos años antes por Pringle (1890) sobre las ninfáceas mexicanas, en el cual, aunque nunca mencionó el nombre de $N$. flavo-virens, distinguía perfectamente $N$. gracilis de $N$. ampla.

Conard además cita en su monografía, el ejemplar colectado e identificado por Pringle (Núm. 3892) como $N$. gracilis Zucc., de una charca artificial cerca de la estación del ferrocarril de El Castillo, a $24 \mathrm{~km}$ aproximadamente al sureste de la ciudad de Guadalajara, diciendo que concuerda bien con el tipo de $N$. flavo-virens.

Existe otro nombre, que nosotros lo hemos encontrado relacionado con este problema, $N$. tussilagifolia Lehm., el cual, Conard solamente lo incluye como un sinónimo de $N$. odorata var. gigantea, probablemente por el comentario que hace Pringle (1897) al describir su viaje por los canales de Xochimilco y decir que en las partes tranquilas del lago, además de $N$. mexicana de flores amarillas él encontró creciendo de manera abundante otra especie de flor blanca, la que él cree probablemente pertenecer a la $N$. tussilagifolia que había sido descrita del cercano lago de Chalco y que no había sido mencionada en su trabajo sobre ninfáceas publicado en 1890 .

Esta es la única explicación que podemos documentar, ya que tanto Planchon (1853) como Lehmann (1853), indican claramente en sus descripciones de $N$. tussilagifolia que el borde de la hoja es ligeramente sinuado y con el ápice del conectivo de los estambres elongado formando un apéndice, características nunca compartidas con el subgénero Castalia al cual pertenece $N$. odorata. Seguramente Conard no vió el ejemplar de Andrieux Núm. 546, por lo cual, incorrectamente incluyó a $N$. tussilagifolia bajo un subgénero que no le correspondía.

De aquí en adelante, diferentes autores al hacer referencia a esta planta endémica de México, lo hacen bajo dos nombres, usándolos indistintamente y siguiendo diferentes criterios, la mayoría de ellos, jamás discutidos. Por ejemplo, Rose (1906), usa el nombre de $N$. gracilis, mientras que Blackaller (1937), Ramírez (1939) y Calderón (1979), entre otros, prefieren utilizar el de $N$. flavo-virens y solamente Henkel et al. (1907) consideran a estos dos nombres pertenecientes a dos especies distintas.

\section{DISCUSIÓN}

A manera de elementos complementarios, se presentan a continuación algunos datos 
tendientes a esclarecer parte de la información documentada en los antecedentes, para situar la discusión en la interpretación correcta que permita distinguir el nombre válido y los nombres que deben quedar como sinónimos.

En primer lugar, es conveniente señalar que si se parte de ejemplares herborizados, resulta difícil distinguir claramente $N$. ampla de $N$. gracilis, especialmente si las hojas son colectadas en estados juveniles. Nuestra experiencia en este caso particular y después de revisar un buen número de ejemplares, nos obligó a tomar en consideración otro tipo de elementos (que resultaron definitivos en su interpretación) que no siempre se incluyen en la etiqueta con datos que acompaña al ejemplar, como lo son, el hábito de la planta, su rango altitudinal, información fenológica, forma y tamaño del rizoma y de las hojas tanto jóvenes como maduras, entre otros. Este otro tipo de información complementaria al ejemplar es especialmente útil en este caso, razón por la cual consideramos conveniente resumirlo en la siguiente tabla.

En la Fig. 2, se observan algunas características morfológicas de estas dos especies, que pueden ayudar en la correcta identificación, especialmente el borde de la hoja, ya que tanto los estilos carpelares como los apéndices del conectivo de los estambres, son muy parecidos y en una misma población pueden variar de tamaño.

La oportunidad de realizar recientemente exploraciones en gran parte del área de distribución de estas especies, nos permitieron contar con material extra de algunas
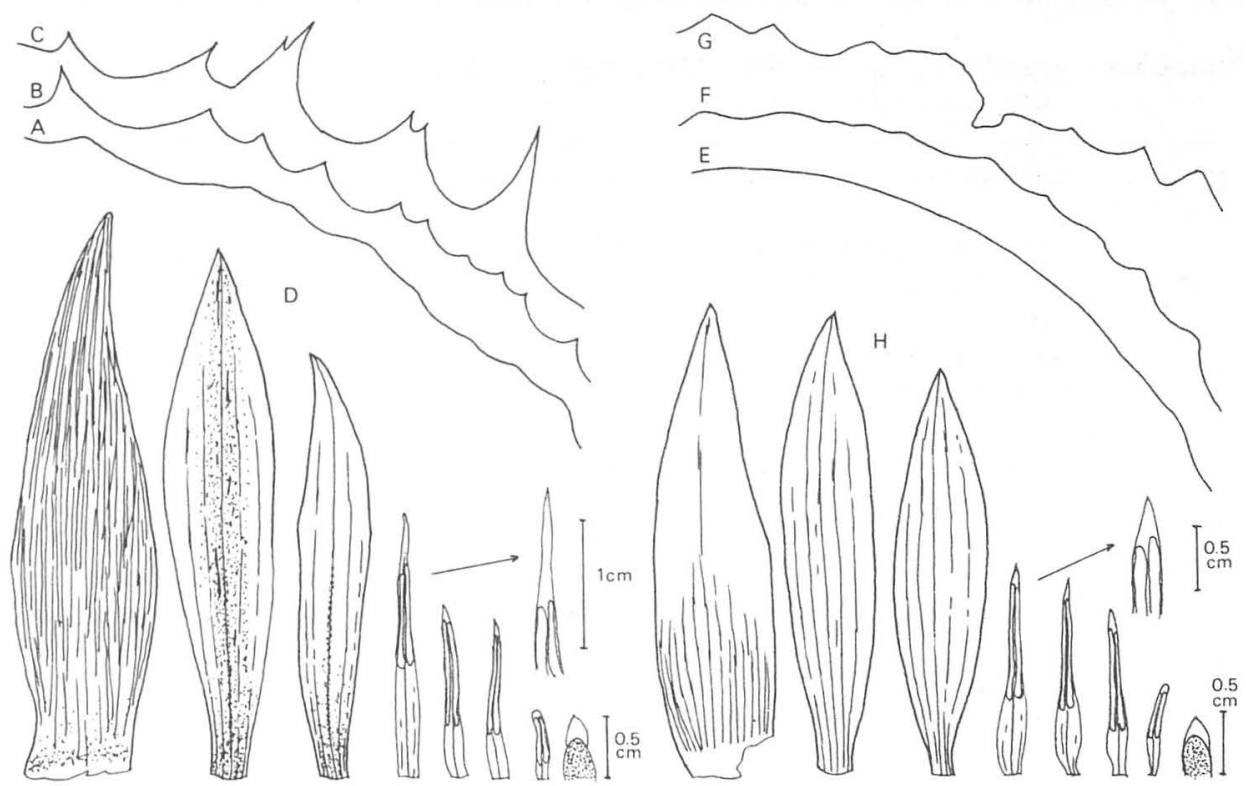

Fig. 2. A-D: Nymphaea ampla. A-C y E-G borde de la hoja, A Langlassé 943; B Lot 2414; C Lot y Novelo 866; D y H partes florales, D Novelo 576; E-H: Nymphaea gracilis. E Lot y Novelo 1017; F Pringle 3891; G y. H Lot y Novelo 1041. Todos los dibujos a $2 / 3$ del tamaño natural, excepto los que tienen escala. 
localidades referidas en los trabajos consultados. De estas visitas, sobresale la localidad cercana a Guadalajara donde Pringle (Núm. 3891) colectó hace casi un siglo la especie en cuestión y donde hoy aún crece en forma abundante. Otra localidad que merece un comentario aparte, es el vaso regulador Espejo de los Lirios en Cuautitlán Izcalli, que puede ser considerado como el último refugio de $N$. gracilis en la Cuenca del Valle de México, cuando antiguamente era un elemento común y aún dominante en relación con las otras especies de Nymphaea.

Con estas colecciones (citadas más adelante) y las observaciones hechas en el campo, las cuales en gran parte están resumidas en la tabla 1, es evidente que $N$. gracilis no pertenece al complejo de $N$. ampla y sus variedades, aunque a simple vista y haciendo solamente el análisis de las hojas se podría pensar lo contrario. De esta forma, por el momento, la variedad speciosa continúa estando restringida a la región caribeña insular y a Sudamérica.

En la consulta de los ejemplares tipo que al parecer estaban relacionados a una sola planta, como son $N$. gracilis Zucc., $N$. flavo-virens Lehm., $N$. undulata Lehm. y $N$. tussilagifolia Lehm. (aunque de esta última no se pudo ver el ejemplar tipo, pero sí se examinó detalladamente la descripción), nos condujo a la conclusión de que pertenecen todas ellas a la misma especie. De acuerdo al artículo 11.3 (prioridad) del Código internacional de nomenclatura botánica, el nombre correcto de esta ninfácea mexicana debe ser $N$. gracilis Zucc. y no $N$. flavo-virens Lehm. como a últimas fechas se venía usando. Por lo tanto, la situación taxonómica de la especie queda de la siguiente manera:

Nymphaea gracilis Zuccarini. Abh. Math. Phys. Cl. Konige Bayer. Akad. Wiss. 1:362. 1832 y en Flora 15(2):74. 1832.

Tipo: México. "In imperio mexicano", Aug. 1827. Karwinski s/n. (HOLOTIPO, M!; fotografía del holotipo de M en MEXU!).

Nymphaea flavo-virens Lehmann. Hamburger Garten-Blumenzeitung 8:370. 1852; también en Ann. Sci. Nat. Bot. ser. III. 19:361. 1853; y en Ind. sem. hort. bot. Hamb. 9. 1852.

Tipo: A partir de una planta cultivada en el Jardín Botánico de Hamburgo (ISOTIPO, $\mathrm{K}$ !; fotografía del isotipo de $\mathrm{K}$ en MEXU!).

Nymphaea undulata Lehmann. Hamburger Garten-Blumenzeitung 9:210. 1853. Tipo: México, Oaxaca. Lacs près Oaxaca, Cordillera. Nov. 1840. H. Galeotti, 4846 (ISOTIPO, K!; fotografía del isotipo de $\mathrm{K}$ en MEXU!).

? Nymphaea tussilagifolia Lehmann. Hamburger Garten-Blumenzeitung 9:530. 1853; también en Ind. sem. hort. bot. Hamb. 10. 1853; y en Ann. Sci. Nat. Bot. ser. IV. 1:326. 1854

Tipo: México, Estado de México. Hab. lacu chalaensi prope Yotla [Ayotla] ditionis Mexici. 14a. mayi. G. Andrieux, 546 (HOLOTIPO, P; no visto).

\section{DISTRIBUCIÓN GEOGRÁFICA}

La distribución geográfica de $N$. gracilis, está restringida a las partes templadas del altiplano, centro y suroeste de México, que abarca los estados de Durango, Aguas- 
TABLA 1. Características diferenciales sobresalientes de Nymphaea ampla var. ampla y de $N$. gracilis

\begin{tabular}{|c|c|c|}
\hline Características & N. ampla var. ampla & $N$. gracilis \\
\hline Borde de la hoja & Sinuoso a dentado & $\begin{array}{l}\text { Entero, sinuoso-dentado a } \\
\text { dentado }\end{array}$ \\
\hline Ápice de los dientes & Generalmente agudos & Obtusos \\
\hline Forma del rizoma & Oblongo & Ovoide \\
\hline Presencia de hojas & Todo el año & Abril a noviembre \\
\hline Nervaduras en el envés & $\begin{array}{l}\text { Muy prominentes } 1 \mathrm{~mm} \text { ó } \\
\text { más }\end{array}$ & $\begin{array}{l}\text { Poco prominentes menos de } \\
1 \mathrm{~mm}\end{array}$ \\
\hline $\begin{array}{l}\text { Ángulo de abertura de los } \\
\text { sépalos }\end{array}$ & Menos de $180^{\circ}$ & $180^{\circ}$ ó más \\
\hline Época de floración & Todo el año & Mayo a noviembre \\
\hline Rango altitudinal & $0 \sim 1500 \mathrm{~m}$ & $\sim 1500$ a $2250 \mathrm{~m}$ \\
\hline
\end{tabular}

calientes, Jalisco, Guanajuato, Michoacán, México, Distrito Federal y Oaxaca y está respaldada por los siguientes ejemplares de herbario:

DURANGO: Laguneta al n de la hacienda de San Juan, Valle de Durango, septiembre 1912, Patoni 524 (ENCB, MEXU); West of Ciudad Durango, September $1^{\text {st }}$. 1951, Maysilles 7661 (MICH). AGUASCALIENTES: 1836, Hartweg 1592 (K). JALISCO: At Capilla de Guadalupe, ca. 10-12 miles east of Tepatitlán, 30, 31 August 1958, McVaugh 17584 (MICH); Guadalajara, octubre 1886, Palmer 283 (GH, K, NY); Laguna de Sayula, Zapotlán, 8 August 1905, Goldsmith 117 (GH); near Guadalajara, 12 October 1891, Pringle 3891 (GH, K, MEXU, MO, NY); La Venta, Zapopan, septiembre 26, 1970, Díaz 1918 (IBUG, MEXU, XAL); Laguna El Castilln (Presa del Ahogado), frente a la estación del ferrocarril San José del Castillo, a $17 \mathrm{~km}$ al sureste de Guadalajara, 20 septiembre, 1979, Lot y Novelo 989 (MEXU); 2 km de Acatlán de Juárez, rumbo a Ciudad Guzmán, 21 septiembre 1979, Lot y Novelo 1014 (MEXU); Laguna de Zapotlán a 3 km al norte de Ciudad Guzmán, 22 septiembre 1979, Lot y Novelo 1017 (MEXU); About 10 miles north Guadalajara, 17 September 1960, Templeton 8872 (ENCB, MICH). GUANAJUATO: Laguna de La Vaca between km 500-510 on road from Leon, Guanajuato to Lagos de Moreno, 30 August 1948, Moore and Wood 4803 (A, MEXU, MICH); 1893, Dugés 301 (GH); 7.8 millas de la iglesia en la Plaza de Dolores, carretera Dolores Hidalgo a Guanajuato, 7 octubre 1977, Kishler 115 (MEXU); Laguna de Yuriria, 24 septiembre 1979, Lot y Novelo 1030 (MEXU); Lago de Yuriria, 25 octubre 1980, Curso Monográfico de Vegetales Acuáticos 543 (ENCB, MEXU, XAL); Borde sur de la Laguna de Yuriria, 11 mayo 1980, Lot y Novelo 1168 (MEXU). MICHOACÁN: Vicinity of Morelia, Rte d'Uruapan, 6 sept. 1910, Arsène 5692 (GH, MO, NY); al oeste del Zapote, Morelia, 28 agosto 1909, Arsène 2403 (GH, K, MEXU, MO); carretera Celaya-Salvatierra km 34, 16 mayo 1965, López y Sánchez s/n (ENCB). MÉXICO: San Pedro Tultepec, municipio de San Mateo Atenco, 3 octubre 1979, Grupo Biología de Campo Intersemestral s/n (FCME); Espejo de Los Lirios, Cuatitlán Izcalli, a 2 km al oeste de 
la autopista México-Querétaro, 21 de agosto de 1979, Lot y Novelo 906 (MEXU); Espejo de Los Lirios, Cuatitlán Izcalli, 2 octubre 1980. Curso Monográfico de Vegetales Acuáticos 531 (ENCB, MEXU, UAMI); Tenayuca, San Bartolo, D. de Tlalnepantla, octubre 1914, Salazar s/n (MEXU); Laguna de Lerma, sin colector (ENCB). DISTRITO FEDERAL: Santa Anita, Valley of Mexico, August 1906, Rose and Rose 11209 (GH, NY); Tacubaya et Mexico, 1865-1866, Bourgeau 5 (GH); Valle de México, 1875. Schaffner 5 (NY); Lago Tláhuac, al norte de Ixtapalapa, septiembre 1936, Blackaller s/n (MEXU). OAXACA: Près Oaxaca, noviembre 1840, Galeotti 4846 (K, MEXU fototipo); Hacienda de Aleman-Etla [?], 3 octubre 1897, Conzatti 500 (GH, MEXU). Otras colectas sin especificar el estado: August 1901, Rose and Hay 6077 (GH, NY); 1834, Andrieux 354 (P); 1827, Berlandier 145 (P); Aug. 1827, Karwinski s/n (M, MEXU fototipo); sin colector, Ex Museo Botanico Berolinensis, ex herb. Caspary, dos ejemplares, probablemente corresponden a isotipos de Nymphaea flavo-virens Lehmann $(\mathrm{K})$; dos ejemplares con una carta que dice que fueron enviados por Mr. Moore, Royal Botanical Gardens, Glasnevin, July 3, 1900 para que figuraran en el Botanical Magazine, aparentemente corresponden al material que fue usado para hacer el dibujo de la lámina número 7781 del Botanical Magazine, no son tipos (K); igual que el anterior solo que con fecha de Dec. 15, 1900 (K).

Al analizar el área de distribución de $N$. gracilis, vemos que probablemente ésta debe ser mayor, debido a las condiciones climáticas en donde normalmente se desarrolla y que comparte en los estados cercanos de San Luis Potosí, Zacatecas y Guerrero.

\section{AGRADECIMIENTOS}

Queremos hacer patente nuestro agradecimiento a los directores y encargados de los siguientes herbarios, que amablemente pusieron a nuestra disposición un total de 62 ejemplares: A, ENCB, FCME, GH, IBUG, K, M, MEXU, MICH, MO, NY, $\mathrm{P}$, XAL y a la Universidad Autónoma Metropolitana, plantel Iztapalapa, México (UAMI) (siglas de acuerdo con Holmgren, Keuken and Schofield, 1981). A los doctores Richard A. Howard y Bernice G. Schubert del Arnold Arboretum, Harvard University, y Jerzy Rzedowski del Instituto Politécnico Nacional por sus atinadas sugerencias durante la elaboración del trabajo y al M. en C. Mario Sousa de la Universidad Nacional Autónoma de México por la revisión crítica del manuscrito. Este trabajo fue apoyado parcialmente con el subsidio del Proyecto PCECNAL 790236 del Programa Nacional Indicativo de Ecología del CONACyT.

\section{BIBLIOGRAFÍA}

BLACKALLER, M.L. 1937. Contribución al estudio de las ninfáceas de los lagos y ciénegas del sur y centro del Valle de México. An. Inst. Biol. Univ. Nal. Autón. México 7:415-455.

CANDOLLE, A.P. DE. 1821. Regni vegetabilis systema naturale 2:39-64.

CASPARY, R. 1878. Nymphaeaceae. En: C.F.P. Martius and A.G. Eichler (Eds.). Fl. Bras. 4(2):129-184.

CONARD, H.S. 1905. The waterlilies. A monograph of the genus Nymphaea. Publ. Carnegie Inst. Wash. (4):1-279.

HENKEL, F., F. REHNELT und L. DITTMANN, L. 1907. Das buch der Nymphaeaceen. Darmstadt. 
HOLMGREN, P.K., W. KEUKEN and E.K. SGHOFIELD. 1981. Index Herbariorum Part I. The herbaria of the world. Edition 7. Dr. W. Junk. B.V., Publishers.

HOOKER, J.D. 1901. Nymphaea flavovirens. Bot. Mag. ser III. 57:tab. 7781.

LEHMANN, J.G.C. 1852. Auszug aus dem Protokolle über die im hamburgischen botanischen Garten zum Blühen gebrachten und daselbst vom Herrn Professor Dr. Lehmann bestimmten Pflanzen. Hamburger Garten-Blumenzeitung 8:369-373.

LEHMANN, J.G.C. 1853a. Ueber die gattung Nymphaea. Hamburger GartenBlumenzeitung 9:193-218.

LEHMANN, J.G.C. 1853b. Zwei neve Arten der gattung Nymphaea. Hamburger Garten-blumenzeitung 9:529-531.

PLANCHON, J.E. 1853. Etudes sur les Nymphéacées. Ann. Sci. Nat. Bot. ser. III 19:17-63.

PRINGLE, C.G. 1890. Notes on the mexican water lilies. Gard. \& Forest 3:415.

PRINGLE, C.G. 1897. Notes of mexican travel XII. My summer in the Valley of Mexico. Gard. \& Forest 10:42-43.

RAMíREZ G, D. 1939. Contribución al conocimiento de la flora acuática del Valle de México. An. Inst. Biól. Univ. Nal. Autón. México 10:33-64.

ROSE, J.N. 1906. The mexican waterlilies. En: Studies in mexican and central american plants V. Contr. U.S. Natl. Herb. 10(3):93-95.

RZEDOWSKI, G.C. DE. 1979. Nymphaeaceae pp. 193-196. En: J. Rzedowski y G.C. de Rzedowski (Eds.). Flora fanerogámica del Valle de México. Vol. I. Editorial Continental.

SALISBURY, R.A. 1806. Description of the natural order of Nymphaeae. Ann. Bot. (Konig \& Sims) 2:69-76.

STAFLEU, F.A. et al. 1978. International Code of Botanical Nomenclature. Regnum veg. 97:1-457.

ZUCCARINI, J.G. 1832. Plantarum novarum vel minus cognitarum, quae in horto botanico herbarioque regio monacensi servantur. Abh. Math.-Phys. Cl. Konige. Bayer. Akad. Wiss. 1:287-396. 